\title{
Control of Stimulated Raman Scattering in the Strongly Nonlinear and Kinetic Regime Using Spike Trains of Uneven Duration and Delay: STUD Pulses
}

\author{
B. J. Albright, ${ }^{1}$ L. Yin, ${ }^{1}$ and B. Afeyan ${ }^{2}$ \\ ${ }^{1}$ Los Alamos National Laboratory, Los Alamos, New Mexico 87545, USA \\ ${ }^{2}$ Polymath Research Inc., Pleasanton, California 94566, USA
}

(Dated: June 9, 2021)

\begin{abstract}
Stimulated Raman scattering (SRS) in its strongly nonlinear, kinetic regime is controlled by a technique of deterministic, strong temporal modulation and spatial scrambling of laser speckle patterns, called Spike Trains of Uneven Duration and Delay (STUD pulses) [B. Afeyan and S. Hüller, Phys. Rev. Lett. (submitted)]. Kinetic simulations show that use of STUD pulses may decrease SRS reflectivity by more than an order of magnitude over random-phase-plate (RPP) or induced-spatial-incoherence (ISI) beams of the same average intensity and comparable bandwidth.

PACS numbers: 52.35.Fp, 52.35.Mw, 52.38.Bv, 52.38.Dx
\end{abstract}

Laser-plasma instabilities (LPI) pose a risk to the realization of laser-driven inertial confinement fusion (ICF) ignition [1. The present approach is to use continuous, ns-time-scale illumination of a target with high-intensity laser beams. However, because of LPI, this may prove to be less than ideal when compared with a novel technique invented by Afeyan [2] employing intermittent, scintillating, space-time illumination which may significantly reduce the levels of nonlinear optical processes. The efficacy of this technique, which employs Spike Trains of Uneven Duration and Delay (STUD pulses), has been demonstrated in the fluid regime of instability evolution from low to moderate gains per speckle, where the linear growth is halted by the use of STUD pulses and any saturation is from pump depletion 2 -4]. This Letter focuses on the application of STUD pulses to Stimulated Raman Scattering (SRS) in settings where kinetic nonlinearity dominates the evolution of driven electron plasma waves (EPW) and where multi-laser-speckle, cooperative behavior can proceed through the exchange of hot electrons and SRS scattered light among laser speckles [5] 7 ]. We find from our initial study that more than order-ofmagnitude reduction in SRS reflectivity can be achieved. The key is to keep SRS growth below the level where secondary, nonlinear processes causing cooperative behavior among hot spots can occur, thus disallowing the self-organized state.

SRS is the resonant, three-wave coupling of a light wave into scattered light and electron plasma waves. It is an LPI process occurring in large amounts in indirectdrive ICF experiments with potentially deleterious effects, including scattering of laser energy out of the hohlraum, redirection of energy within the hohlraum, and generation of hot electrons that may contribute to capsule preheat. At the National Ignition Facility (NIF), experiments show $\sim 50 \%$ inner-cone beam energy loss to SRS [1. Laser facilities such as OMEGA and the NIF employ beam smoothing, whereby random phase plates (RPP) break the laser beams into "speckles" to effect a quasi-uniform (on the large scale) intensity pro- file across the beam, though introducing into the beam small-scale, high-intensity variations or "speckles." In vacuum, speckles have characteristic size $8 f^{2} \lambda_{0}$ (longitudinally) by $f \lambda_{0}$ (transversely), where $f$ is the optical focal parameter and $\lambda_{0}$ is the laser wavelength. The scaling of SRS reflectivity $R_{S R S}$ with laser intensity $I$ in a solitary speckle in plasma has been measured [8] and found in the electron trapping regime $k \lambda_{\text {De }} \gtrsim 0.3$ ( $k$ is the EPW wave number and $\lambda_{\mathrm{De}}=\sqrt{k_{B} T_{e} / 4 \pi n_{e} e^{2}}$ is the Debye length for plasma of electron density $n_{e}$ and temperature $T_{e}$ ) to behave nonlinearly, increasing sharply at a threshold intensity $I_{\mathrm{th}}$ and saturating for $I>I_{\text {th }}$. The physics in this regime is governed by the growth of large-amplitude EPW that trap "resonant" electrons with speeds along the wave propagation direction matching the wave's phase speed; this reduces local Landau damping 9, enhances instability growth, and lowers the EPW frequency [10. At high intensity, trapping introduces variation in EPW phase velocity across the speckle and causes wave phase fronts to bend 11 14. As EPW grow to large amplitude, secondary, nonlinear processes have been proposed to break the phase fronts into small-transverse-scale filaments $14-16$ that further contribute to nonlinear saturation. An effect of saturation, observed in simulations [5], is the generation of hot electrons and back- and side-scattered light waves that propagate obliquely out of hot spots and enhance SRS growth in neighboring speckles through larger SRS seed levels and reduced EPW Landau damping. At high gain in two spatial dimensions (2D), this coupling enables networks of speckles to self-organize [5] and exhibit emergent behavior where reflectivity exceeds that of the sum of contributions from individual, non-interacting speckles. The nonlinear nature of SRS in this regime is robust, with a threshold at modest laser intensity, $\gtrsim 10^{14} \mathrm{~W} / \mathrm{cm}^{2}$ for NIF laser conditions where $k \lambda_{\mathrm{De}} \approx 0.3$ and the highest levels of backscatter are found [17].

Our intent here is to show that the use of STUD pulses [2], effective for controlling LPI over long time scales in the fluid regime of instability evolution [2 4], 
may also inhibit EPW growth in the highly nonlinear, kinetic regime. STUD pulses deliver laser power in a sequence of pulses or spikes on the instability growth or hot spot crossing time scale (sub-ps, typically, for SRS) with randomized laser speckle patterns in between one or more successive spikes. By introducing on-off sequences of pulses and by spatially scrambling locations of hot spots, reinforcing processes within a hot spot and the interconnectivity between hot spots that leads to large instability growth are disrupted. STUD pulses introduce degrees of freedom that can be optimized [2]. These include the ratios $L_{H S}: L_{\mathrm{INT}}: L_{\text {spike, }}$, where the interaction length is $L_{\mathrm{INT}}=4 L_{n}\left[\alpha^{2} I_{14}+\left(\nu_{2} / \omega_{2}\right)^{2}\right]^{1 / 2}$. Here, the density scale length is $L_{n}=\left|\nabla \log n_{e}\right|^{-1}, I_{14}$ is intensity in $10^{14} \mathrm{~W} / \mathrm{cm}^{2}, \nu_{2}$ and $\omega_{2}$ are the local Landau damping rate and frequency of the EPW, respectively, $\alpha^{2}=1.42 \lambda_{0}^{[\mu m]} L_{n}^{[100 \mu m]}\left|V_{1} / V_{2}\right|\left(n_{e} / n_{\mathrm{cr}}\right)^{-1}$, and $V_{1} / V_{2}$ is the ratio of group velocities of scattered SRS light to the EPW [2]. Spike length $L_{\text {spike }}$ is the distance traveled by scattered light during the 'on' time $\tau_{\text {spike }}$ and $L_{H S} \sim 4 f^{2} \lambda_{0}=90 \mu \mathrm{m}$ is the characteristic size of a hot spot in our plasma. Other degrees of freedom are duty cycle (the ratio of $\tau_{\text {spike }}$ to on+off time), the spatial scrambling rate $\times n_{\text {scram }}$ (how many identical spikes before the RPP pattern changes) and the "jitter" (random variation of each $\tau_{\text {spike }}$; all calculations in this Letter used $0 \%$ jitter). Hence, " $5000 \times 1,1: 1: 1 / 2$ " indicates a STUD pulse sequence with $50 \%$ duty cycle, $0 \%$ jitter, and a spike duration half as long as a hot spot crossing time in plasma where the time to cross $L_{\mathrm{INT}}$ for the three-wave process is comparable to that of crossing the hot spot. Most of the results we present are for cases $5000 \times 1,1: 1: 1 / 2$ and $1: 1: 1$ in strong to very strong nonlinear kinetic regimes (SRS gains of $6-11$ at the average intensity). Note that configurations where 'on' time is much greater than 'off' time, e.g., $8000 \times 1$ or $9500 \times 1$, resemble the ISI model of beam smoothing [18] at the same bandwidth.

To understand better the behavior of STUD pulses in the trapping regime, we have run VPIC particle-incell simulations [19] of a 2D plasma of size $500 \times 80 \mu \mathrm{m}$ in $(x, z)$, with a laser beam polarized along $y$ launched at $x=0$ as described in Ref. 6]. The laser has wavelength $\lambda_{0}=0.351 \mu \mathrm{m}$ and an RPP speckle pattern for $f \backslash 8$ speckles is used, approximating a NIF inner-cone beam in hohlraum plasma. The density has a gradient along $x$ with $n_{e}=0.12 n_{\text {cr }}$ at the center, changing from $\pm 0.013 n_{\text {cr }}$ to $\pm 0.03 n_{\text {cr }}$ across the box, comparable to the $L_{n} \sim \mathrm{mm}$ encountered in NIF ignition hohlraums in regions of high SRS backscatter [17]. Taking $\nu_{2}=\nu_{2}^{\mathrm{Max}}$, as for Maxwellian plasma, in the $k \lambda_{\text {De }} \approx 0.3$ regime yields $L_{\mathrm{INT}} \sim 95-99 \mu \mathrm{m}$ for the range of intensities simulated. We use $36864 \times 4096$ cells $\left(\Delta x=1.2 \lambda_{\text {De }}\right.$ and $\left.\Delta z=1.7 \lambda_{\mathrm{De}}\right)$ and $256-512$ electron macroparticles/cell; ions are stationary. The electrons have $T_{e}=2.6 \mathrm{keV}$ $\left(k \lambda_{\mathrm{De}}=0.3\right)$. The STUD pulse speckle patterns are

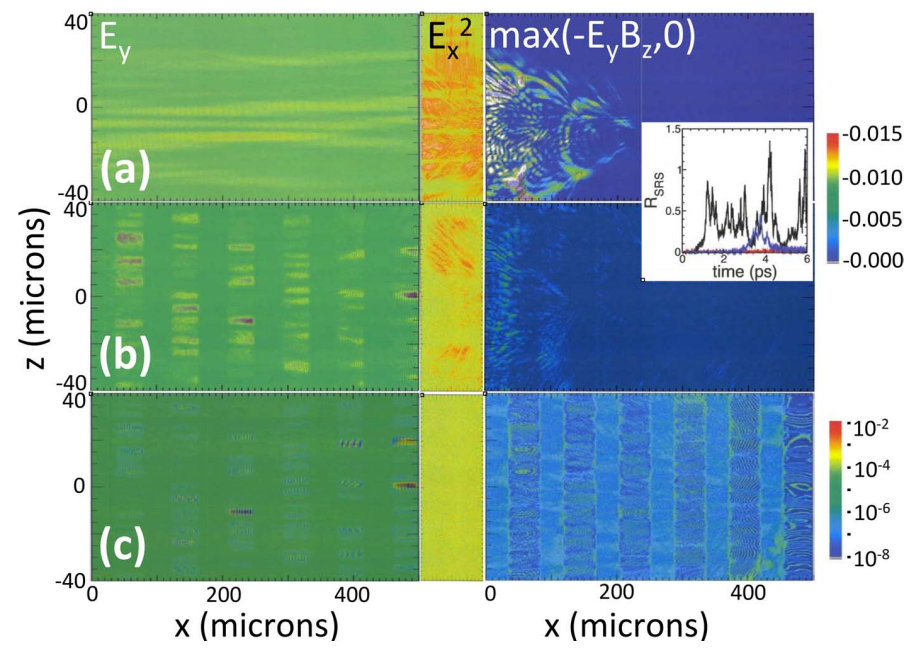

FIG. 1. (Color) $E_{y}$ (left) and corresponding instantaneous backscattered Poynting flux $\max \left(-E_{y} B_{z}, 0\right)$ (right) over the 2D simulation volume for three cases: (a) an RPP laser beam at average laser intensity $\langle I\rangle=5 \times 10^{14} \mathrm{~W} / \mathrm{cm}^{2}$ (top), (b) a $5000 \times 1$, 1:1:1/2 STUD pulse beam at time-averaged incident laser intensity $\langle I\rangle=5 \times 10^{14} \mathrm{~W} / \mathrm{cm}^{2}$ (center); and, (c) the same STUD pulse beam, but with $\langle I\rangle=3.2 \times 10^{14} \mathrm{~W} / \mathrm{cm}^{2}$ (bottom) (note logarithmic scale on Poynting flux). The center panels are electrostatic field energy $E_{x}^{2}$ for the leftmost $80 \mu \mathrm{m}$ of each simulation, showing EPW wave amplitude correlated with instantaneous SRS backscattered Poynting flux. The inset is reflectivity vs. time for cases (a) (black) and (b) (blue); (c) (red) evinces negligible backscatter. The times shown are 1.6 ps (a) and 3.6 ps (b,c), chosen when large, bursts of SRS backscatter were present in (a) and (b).

generated from pre-computed RPP phases for a wide beam, sampling $80-\mu \mathrm{m}$, non-overlapping segments for each STUD pulse. Each simulation employs the same sequence of speckle patterns to within an overall intensity modulation, allowing variation of intensity, duty cycle, and modulation period. (Statistical variation was assessed by altering the sequences of STUD pulses; $\sim 10 \%$ relative $R_{S R S}$ variation was found in a range of cases considered.) The simulation boundaries absorb electromagnetic waves and reinject electrons as Maxwellian at initial temperature $T_{e}$. The simulations were run until apparent "steady-state" in time-averaged $R_{S R S}, 10-20$ ps.

Fig. 1 shows a comparison of three simulations: (a) (top row) is for an RPP beam with $\langle I\rangle=5 \times$ $10^{14} \mathrm{~W} / \mathrm{cm}^{2}(G=11)$; (c) (bottom) is for a STUD pulse beam of time-averaged laser intensity $\langle I\rangle=3.2 \times$ $10^{14} \mathrm{~W} / \mathrm{cm}^{2}(G=7.5)$. Linear SRS gains $G$ are computed from $G=4 \pi\left(\gamma_{0} / \omega_{0}\right)^{2}\left(2 \pi L_{n} / \lambda_{0}\right) g\left(n / n_{c}\right)^{-1}(1-$ $\left.\nu_{1} \nu_{2} / \gamma_{0}^{2}\right)$, where $\left(\gamma_{0} / \omega_{0}\right)=0.0043 \sqrt{I_{14}} \lambda_{0}^{[\mu m]}, \nu_{1}$ is the damping rate of the daughter light wave, and $g(n) \equiv$ $\sqrt{1-2 \sqrt{n}}[(1 / \sqrt{n})-1]$ [2]. Accounting for backscatter loss, (a) and (c) have comparable net time-averaged power incident on the left boundary, though (c) has only $64 \%$ of the incident time-averaged laser power. Case (b) (center) is for a STUD pulse beam at the same time-averaged incident laser intensity as (a): $\langle I\rangle=5 \times$ 


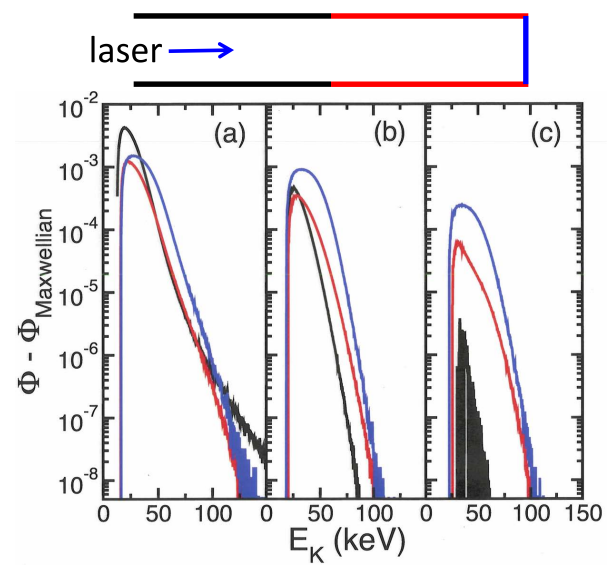

FIG. 2. (Color) Hot electron flux per unit length $\Phi$ vs. electron energy $\left(E_{K}\right)$ for the three simulations in Fig. 1. Shown are trapped particle fluxes, obtained by subtracting contributions from a Maxwellian. Fluxes are measured on boundary regions, as indicated by the colors (c.f. the simulation box above, drawn to scale): $z= \pm 40 \mu \mathrm{m}, 0<x<250 \mu \mathrm{m}$ (black); $z= \pm 40 \mu \mathrm{m}$, $250<x<500 \mu \mathrm{m}$ (red) and $x=500 \mu \mathrm{m}$ (blue).

$10^{14} \mathrm{~W} / \mathrm{cm}^{2}(G=11)$. The leftmost panels show $E_{y}$ (or the vacuum speckle pattern for the RPP case). The rightmost panels are instantaneous backscattered Poynting flux $\max \left(-E_{y} B_{z}, 0\right)$. Case (a) evinces continual bursts of self-organized backscatter with peak $R_{S R S}>1$. In (c), no self-organization is seen in backscattered light or longitudinal electric field. Case (b) is intermediate, showing quiescent periods of low backscatter punctuated by occasional episodes of partial self-organization when largeamplitude speckles $(I \gtrsim 10\langle I\rangle)$ exhibit large-amplitude EPW and secondary processes, such as obliquely sidescattered light, occur at sufficient amplitude to seed SRS in otherwise stable regions of plasma (seen in the finite backscattered SRS Poynting flux across the left of the box). The instantaneous $R_{S R S}$ at the left boundary is shown in the inset for cases $(\mathrm{a}-\mathrm{c})$ (black, blue, and red curves, respectively); the times plotted are $1.6 \mathrm{ps}$ for the RPP (during the first large SRS burst), and 3.6 ps for the STUD pulse simulations [during the first, large SRS burst in (b)]. The central panels are $E_{x}^{2}$ over the leftmost $80 \mu \mathrm{m}$ of the volume and indicate EPW amplitude correlated with the large bursts of SRS in (a) and (b).

In Fig. 2, we compare for cases (a-c) time-integrated hot electron flux per unit area exiting the simulation. The black curves are fluxes leaving the $\pm z$ boundaries from the left half of the simulation volume, the red curves, leaving $\pm z$ from the right of the volume, and the blue curves, leaving from the $+z$ boundary. Prior work showed that large fluxes of tail electrons leaving the left of the volume (i.e., large black curves) are signatures of large-amplitude EPW with ensuing nonlinear self-focusing and filamentation and, ultimately, collective behavior among speckles [6, 7]. The three cases evince elevated distribution function tails as a consequence of

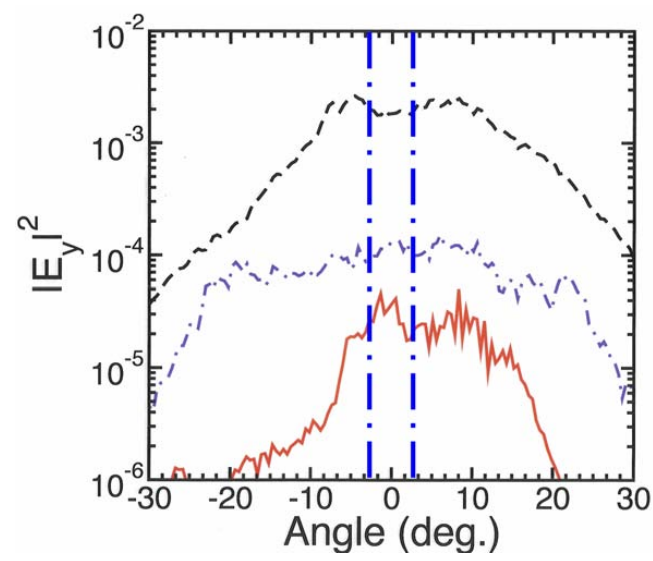

FIG. 3. (Color) Angular distribution of the time-averaged backscattered light power for case (a) (black, dashed), (b) (dotdashed, blue), and (c) (red) as in Figs. 1 and 2. The spectra for (b) and (c) evince lower backscattered light power, but at finite angle with respect to the incident laser (cone angle $|\theta|<1 / 2 f$, shown by the vertical lines).

trapping, though the RPP case traps not only far more tail electrons $[60 \times$ more than $(\mathrm{c}), 6 \times$ more than $(\mathrm{b})]$, but also shows far more side-scattered hot electrons exit nearest the laser entrance; moreover, hot electrons at very high energy $\left(E_{K}>100 \mathrm{keV}\right)$ are present (absent for the STUD pulse beams). The use of STUD pulses has therefore decreased the number of hot electrons exchanged laterally among laser speckles, a key part of inter-speckle self-organization [7] and a possible contributor to capsule preheat in ICF experiments. In Fig. 3, we compare the angular spread of SRS light for the same cases as above. The use of STUD pulses leads to a dramatic overall reduction in SRS power (and hence, amplitude of the SRS seed in neighboring speckles). As with the $\mathrm{RPP}$, the angular spread is finite, with most of the power falling outside the incident laser cone $|\theta|<1 / 2 f$ shown by the vertical lines. While the existence of coherent, oblique cones of backscattered light is not unique to this nonlinear regime - indeed, they appear in paraxial models with diffraction 2, 20 —additional side-scatter results from trapping and EPW filamentation [5, 16, that is absent in fluid models; the use of STUD pulses reduces dramatically the levels of such side-scatter.

Finally, in Fig. 4, we show the dependence of $R_{S R S}$ on time-averaged incident laser intensity (left) and linear gain at the average intensity (right) for RPP and STUD pulses over a range of plasma and laser conditions. The use of STUD pulses reduces dramatically $R_{S R S}$ compared with RPP and ISI-like beams with the same time-averaged laser power. This is so even in cases of very high linear gain. As seen from comparison of the $R_{S R S}$ from the ISI-like points (the $8000 \times 1,1: 1: 1 / 2$ and $9500 \times 1,1: 1: 1 / 2)$ and $5000 \times 1,1: 1: 1 / 2$ cases, "healing time" is key: it is not enough to simply add bandwidth and spatial scrambling. By optimizing this healing 
time for given "on+off" time and time-averaged power, STUD pulses may be optimized to significantly outperform ISI. From comparison of the $5000 \times 4,1: 1: 1 / 2$ and the $5000 \times 1,1: 1: 1 / 2$ cases, we find that spatial scrambling of the locations of the hot spots is also necessary to avoid effects of recurrence and correlation among successive hot spots [21. Also, for fixed "on+off" time and time-averaged power, lengthening 'off' time requires shortening $\tau_{\text {spike }}$ and increasing the average speckle intensity correspondingly. Taken to an extreme, this can lead to enhanced trapping and associated EPW nonlinearity, evidenced by the $2000 \times 1$ datum shown in Fig. 4 [which also has significant hot electron sidescatter (not shown) compared with the $5000 \times 1,1: 1: 1 / 2$ case at the same average power].

Examination of velocity distribution functions and EPW amplitudes shows strong trapping and only modest EPW damping between cycles. This trapping modifies $L_{\mathrm{INT}}$ and suggests that possible threshold behavior may arise when $L_{\text {spike }}$ becomes less than $L_{\mathrm{INT}}$ and when SRS goes from strong to weak damping. Consider the two $5000 \times 1$ and the $9500 \times 1$ STUD pulse cases at the highest intensity $(G=11)$. In the former, SRS in the largest amplitude hot spots $(I \gtrsim 10\langle I\rangle)$ would be in the weak damping limit (WDL) if one applies the inferred $\nu_{2}$ from simulations $\left(\approx 0.1 \nu_{2}^{\operatorname{Max}}\right)$, and $L_{\mathrm{INT}} \approx 120 \mu \mathrm{m}$. The 1:1:1/2 STUD pulse case, with the lowest $R_{S R S}$, has $L_{\text {spike }} \sim 0.37 L_{\text {INT }}$ for these maximal speckles, i.e., STUD pulses much shorter than $L_{\mathrm{INT}}$. In contrast, the 1:1:1 case has $L_{\text {spike }} \sim L_{\mathrm{INT}}$. In the $9500 \times 1,1: 1: 1 / 2$ ISI-like case, while $L_{\mathrm{INT}}=0.5 L_{\text {spike }}$, reduction of $\nu_{2}$ causes the SRS to go to the WDL for an average intensity speckle, with correspondingly large $R_{S R S}$.

We have shown that SRS reflectivity may be lowered by more than an order of magnitude with the use of properly designed STUD pulses in settings where EPW trappinginduced nonlinearity is prevalent. This reduction stems from arresting large-amplitude EPW that give rise to cooperative behavior among laser speckles through the exchange of hot electrons and backscatter SRS waves, thus disallowing their self-organization. The substantial promise and generality of the STUD pulse technique 2. to a range of settings, including SRS in the strongly nonlinear, trapping regime considered here, would seem to impel serious consideration for how STUD pulses might be achieved in future ICF laser systems such as the Green option on the NIF or next-generation high-repetition-rate laser systems.

Work performed under the auspices of the U.S. Dept. of Energy under contract W-7405-ENG-36. BJA \& LY were supported by DOE NNSA ICF and LDRD Programs and the DOE NNSA-OFES Joint program in HEDLP. BA's work was supported by grants from the DOE NNSA-OFES Joint program in HEDLP and by SBIR grants from OFES. Simulations were performed on ASC Roadrunner and Cielo. We acknowledge use-

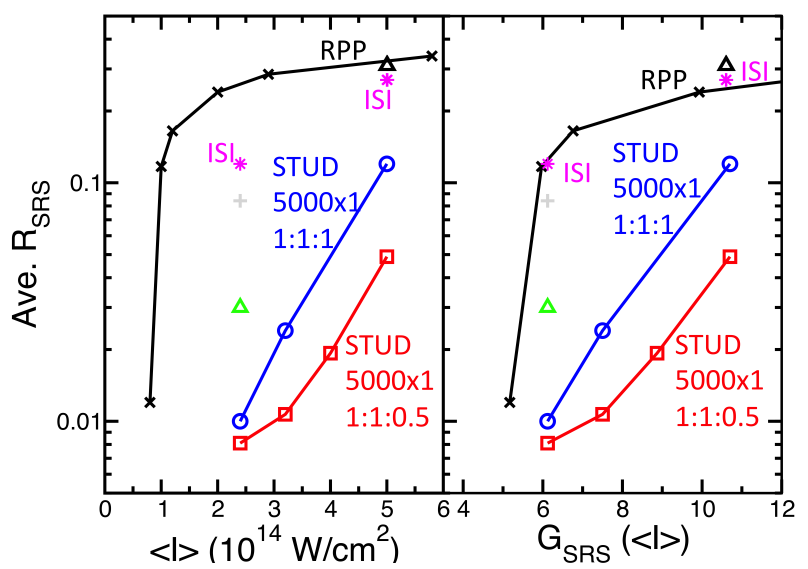

FIG. 4. (Color) SRS reflectivity plotted as a function of average incident intensity (left) and linear gain (right) for RPP and STUDpulse beams for a variety of laser and plasma conditions, showing the efficacy of STUD pulses for reducing $R_{S R S}$. The black points (marked $\times$ and $\triangle$ ) are for RPP beams for two different density variations across the box in $x: \pm 0.013 n_{\mathrm{cr}}$ and $\pm 0.03 n_{\mathrm{cr}}$, respectively. The red ( $\square$ ) and blue (०) curves are for STUD pulses with $5000 \times 1,1: 1: 1 / 2$ and $5000 \times 1,1: 1: 1$ (i.e., twice the "on" time) for the latter density profile. The magenta datapoints labeled "ISI" are for STUD pulses with $8000 \times 1,1: 1: 1 / 2$ (left) and $9500 \times 1,1: 1: 1 / 2$ (right) and indicate little advantage over RPP for the conditions shown. The green $(\triangle)$ is for STUD pulses with $5000 \times 4,1: 1: 1 / 2$, i.e., scrambling every 4 pulses. The $(+)$ is for $2000 \times 1,1: 1: 1 / 2$.

ful discussions with S. Hüller, J. Garnier, J. Fernández, D. Montgomery, J. Kline and S. Batha.

[1] William Goldstein and Robert Rosner, eds., Proc. Science of Fusion Ignition on NIF Workshop, LLNL-TR570412, San Ramon, CA, Lawrence Livermore National Security, LLC, (2012); William L. Kruer in Laser-Plasma Interactions and Applications, edited by P. McKenna et al., Scottish Graduate Series (Springer, Switzerland, 2013).

[2] B. Afeyan and S. Hüller, "Optimal Control of LaserPlasma Instabilities using Spike Trains of Uneven Duration and Delay (STUD Pulses)," Phys. Rev. Lett. (submitted); also arXiv:1304.3960.

[3] B. Afeyan and S. Hüller, Europ. Phys. J. Web of Conferences (in press); also arXiv:1210.4462.

[4] S. Hüller and B. Afeyan, Europ. Phys. J. Web of Conferences (in press); also arXiv:1210.4480

[5] L. Yin, B. J. Albright, H. A. Rose, K. J. Bowers, B. Bergen and R. K. Kirkwood, Phys. Rev. Lett. 108, 245004 (2012).

[6] L. Yin B. J. Albright, H. A. Rose, K. J. Bowers, B. Bergen, R. K. Kirkwood, D. E. Hinkel, A. B. Langdon, P. Michel, D. S. Montgomery, J. L. Kline, Phys. Plasmas 19, 056304 (2012).

[7] L. Yin, B. J. Albright, H. A. Rose, D. S. Montgomery, J. L. Kline, R. K. Kirkwood, P. Michel, K. J. Bowers, B. Bergen, Phys. Plasmas 20, 012702 (2013).

[8] D. S. Montgomery, J. A. Cobble, J. C. Fernández, R. J. Focia, R. P. Johnson, R. Renard-LeGalloudec, H. A. 
Rose, and D. A. Russell, Phys. Plasmas 9, 2311 (2002).

[9] T. O'Neil, Phys. Fluids 8, 2255 (1965).

[10] G. J. Morales, and T. M. O'Neil, Phys. Rev. Lett. 28, 417 (1972).

[11] L. Yin, B. J. Albright, K. J. Bowers, W. Daughton, and H. A. Rose, Phys. Rev. Lett., 99, 265004 (2007).

[12] L. Yin, B. J. Albright, H. A. Rose, K. J. Bowers, B. Bergen, D. S. Montgomery, J. L. Kline, and J. C. Fernández, Phys. Plasmas 16, 113101 (2009).

[13] J. W. Banks, R. L. Berger, S. Brunner, B. I. Cohen, and J. A. F. Hittinger, Phys. Plasmas 18, 052102 (2011).

[14] C. Rousseaux, S. D. Baton, D. Bénisti, L. Gremillet, J. C. Adam, A. Héron, D. J. Strozzi, and F. Amiranoff, Phys. Rev. Lett. 102, 185003 (2009).

[15] H. A. Rose, Phys. Plasmas 12, 12318 (2005); H. A. Rose and L. Yin, Phys. Plasmas 15, 042311 (2008).

[16] P. E. Masson-Laborde, W. Rozmus, Z. Peng, D. Pesme, S. Hüller, M. Casanova, V. Yu. Bychenkov, T. Chapman, and P. Loiseau, Phys. Plasmas 17, 092704 (2010).

[17] R. K. Kirkwood et al., Phys. Plasmas 18, 056311 (2011); D. E. Hinkel et al., Phys. Plasmas 18, 056312 (2011); S. H. Glenzer et al., Phys. Rev. Lett. 106, 085004 (2011).
[18] R. Lehmberg and J. Rothenberg, J.Appl. Phys. 87, 1012 (2000).

[19] K. J. Bowers, B. J. Albright, L. Yin, B. Bergen, and T. J. T. Kwan, Phys. Plasmas 15, 055703 (2008); K. J. Bowers, B. J. Albright, B. Bergen, L. Yin, K. J. Barker, and D. J. Kerbyson, Proceedings of the ACM/IEEE conference on Supercomputing, Austin, 2008 (Piscataway, NJ, USA: IEEE Press) pp 1-11; K. J. Bowers, B. J. Albright, L. Yin, W. Daughton, V. Roytershteyn, B. Bergen, and T. J. T. Kwan, J. Phys.: Conf. Ser. 180, 012055 (2009).

[20] T. Kolber, W. Rozmus, and V. T. Tikhonchuk, Phys. Fluids B, 5, 138 (1993).

[21] Because of how geometry affects multi-speckle coupling, transverse cross-speckle coupling through SRS side-loss hot electrons has been shown to be lower in $3 \mathrm{D}$ vs. $2 \mathrm{D}$ [7]. Moreover, the probability of spatial recurrence of hot spots is vastly smaller in $3 \mathrm{D}$ than in $2 \mathrm{D}$. As a consequence, the advantages of STUD pulses in this nonlinear kinetic regime of SRS are expected to be more pronounced in more realistic, 3D geometry. 